\title{
ONTOLOGY-BASED RULE COMPLIANCE CHECKING FOR SUBSURFACE OBJECTS
}

\author{
C. Métral ${ }^{1 *}$, V. Daponte ${ }^{1}$, A. Caselli $^{1}$, G. Di Marzo ${ }^{1}$, G. Falquet $^{1}$, \\ ${ }^{1}$ University of Geneva, Switzerland \\ (claudine.metral, vincenzo.daponte, ashley.caselli, giovanna.dimarzo, gilles.falquet)@unige.ch
}

Commission VI, WG VI/4

KEY WORDS: Rule compliance checking, Ontology, 3D city model

\begin{abstract}
:
This paper presents a model for representing compliance rules related to subsurface objects. Rules expressed in this model can be automatically evaluated (using SHACL or SPARQL) on existing 3D city models expressed in RDF. The main characteristics of the proposed model are (1) its expressiveness, that comes from the use of formal ontologies for representing the rules and the objects they refer to, (2) its integrative nature, given by the interconnection among the proposed ontologies and the connection of these ontologies with CityGML and IFC (in an ontological form), and (3) its multi-geometry aspect. Preliminary results allow to automatically evaluate formally expressed compliance rules for underground objects in a 3D city model, that will considerably ease the task of professionals of the field.
\end{abstract}

\section{INTRODUCTION}

\subsection{D city models and underground objects}

Cities contain many and various subsurface objects, such as utility networks, basements of buildings, tree roots, groundwater, or archaeological remains. Over the last years the interest in underground objects has led to the development of $3 \mathrm{D}$ models to represent these man-made or natural objects. These models, such as the CityGML Utility Network ADE, generally complement the standard CityGML model that was initially developed to represent surface or above surface objects.

\subsection{Rule compliance checking for subsurface objects}

Rule compliance is based on legal texts that stipulate the spatial configurations between objects and their surroundings, in order to avoid interferences or to specify safety rules. Without compliance, inadequately designed utilities will continue to exist or will be constructed. A rule compliance checking mechanism is thus needed to help identify non-compliances and limit the negative impact of such malfunctions (Xu and Cai, 2020).

Using 3D city models, rule compliance checking means spatial compliance checking between 3D representations of the involved objects. Rules have thus to be expressed in a form compatible with such 3D representations. In particular, 3D spatial relationships must be defined, for example, to express that the telecommunication and the electricity networks should be located below the sidewalk at a depth of 0.4 meter, followed respectively by the gas network ( 0.8 to 1 meter depth) and the water network (1.2 to 1.5 meter depth), and that the waste water network (1.6 meter depth) is generally located under the middle of the roadway. The 3D objects associated to such rules are below, on or even above ground. They have to be described with their semantic properties and their geometry.

\subsection{An ontology-based approach}

The issue addressed in this paper is how to automatically test if the subsurface objects represented in a 3D city model of a given area comply with the rules defined in legal texts. This implies to define a rule model that (1) is sufficiently close to the vocabulary of the domain specialists to facilitate the human or machine translation of the rules to a formal expression and (2) has a formal semantics.

We propose a formal model to represent the rules and a set of interconnected OWL ontologies to represent the subsurface objects as well as their spatial relationships and their geometry. The proposed rule model is close to the natural language expression of the rules in the sense that most of the concepts that appear in the legal texts (subsurface objects, spatial relations, constraints, ...) can be directly mapped to concepts of the model. Therefore, the model can be directly used by an information system engineer or a geomatician to express a large variety of existing rules. It can also serve as a target model for the automated translation of the rules from natural language to a formal expression. Since the model has a formal semantics the rules can be automatically transformed to executable SHACL rules or SPARQL queries that can be executed on an RDF triplestore. The proposed model has been successfully used to represent official rules on real datasets from SITG (the information system of Geneva territory).

\section{RELATED WORK}

As depicted by Pauwels et Zhang (2015), there exists three main approaches to tackle the modelling of regulation knowledge issues within a semantic regulation compliance checking process: (i) hard-coded rules, (ii) rules expressed as queries, and (iii) usage of dedicated rule languages.

Among the query-based approaches Wagner et al (2013) propose a technique based on OCL (the UML Object Constraint Language) expressions to validate the geometry of CityGML models. This approach is well suited for checking universal and stable rules (e.g. the points in a polygon must be coplanar) that must be true in every context. Similarly, the INTERLIS modelling language has been used to represent higher level constraints in legal 3D spaces (Kalogianni et al, 2017).

A comprehensive approach to semantic compliance of underground utilities has been designed and developed by $\mathrm{Xu}$ 
and Cai, 2020). The authors present a framework for: (i) integrating spatial data about underground utility networks (through a utility product ontology, a transportation object ontology, and a geometry ontology); (ii) representing spatial constraints about utility objects with the USRO ontology; and (iii) evaluating constraints expressed in natural language. This work mainly focuses on giving the non-IT professionals the possibility to enter compliance rules in natural language and then automatically check them. A natural language parser maps the textual expression of the constraints to objects of the USRO model. The USRO model is semi-formal in the sense that distances, distance restrictions, landmark objects, trajector objects, spatial indicators, etc. are represented by strings (extracted from the natural language expression). The rule evaluation requires a mapping phase to map these strings to the corresponding objects of the utility and transportation ontologies because the vocabulary employed by the user may differ from the vocabulary of these ontologies. Once the correspondences have been established (using synonym lists and auxiliary terminologies) the rules can be translated to GeoSPARQL queries and executed on the data. Since the natural language parsing and the mapping phases are not $100 \%$ accurate, one can say that this approach eases the use at the expense of complete accuracy.

3D Spatial relations play an important role in the representation of rules and regulations for subsurface objects (whereas the rules for surface objects generally refer to 2D spatial relations). These relations have long been studied from a mathematical point of view, in geometry and topology. Over the last decades this knowledge has been transferred to knowledge representation models, such as formal ontologies, that are suitable for automated processing.

The conceptual framework presented by Clementini and Laurini (2008) describes spatial relationships in terms of topological, projective, and metric ones. It also takes into account the cardinality of the spatial relationships (the number of geometrical objects that participate in the relationship), the granularity of the relationships and the dimension of the geometrical objects involved. Bucher et al (2010) review spatial properties and relations relevant to the management of semantics in city models. They use a description logic formalism (ontology) to represent the relations and properties that have been considered as important for city models. This ontology should support an application designer making choices in terms of data and algorithms selection. More recently ontologies have been used for representing the semantic, spatial and temporal features of an environment for robot task planning (Gayathri and Uma, 2018). The choice of ontologies enables in particular spatial reasoning, that means reasoning about spatial objects in the environment.

\section{PROPOSED APPROACH}

\subsection{Ontology-based approach}

The proposed rule modelling technique is based on the development and interconnection of a set of ontologies that precisely represent the concrete and abstract concepts that appear in compliance rules. These ontologies play three roles: 1) they provide an integration schema to collect geodata from different sources and integrate them in a single RDF graph;2) they form the vocabulary with which the compliance rules can be expressed; 3) the axioms of these ontologies are used by the SHACL or SPARQL evaluation engines to infer implicit knowledge during the rule checking phase (Figure 1).

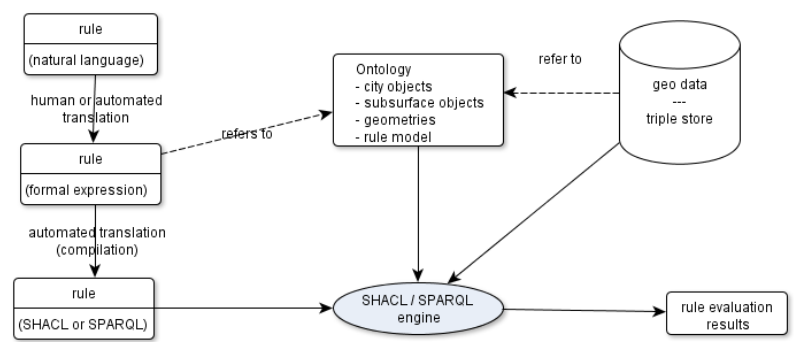

Figure 1. Rule translation and execution

\subsection{Methodology}

\subsubsection{Rule Model}

The rule model has been designed through the following steps. At first, the regulations issued by several Swiss entities that involve subsurface objects have been analyzed. The analysis focused on their structure, rather than their content. During the second step, the rules have been split into basic components. These components have been analyzed and abstracted into higher level classes. Once identified, the classes have been merged into a unified model to represent compliance rules. Even if starting from the Swiss model we propose a generic model obtained by extracting universal parts that are represented by high level classes.

\subsubsection{Rule vocabulary}

Rule content has been analyzed in order to identify the associated vocabulary. This vocabulary has been organized in different ontologies (geometry, spatial relations) interconnected with the main ontology (subsurface objects). Existing vocabulary in standards has also been identified, leading to connections with IFC and CityGML, as well as with the CityGML Utility Network ADE

\subsubsection{Validation}

The evaluation of this model relies on assessing its expressive capacity against a wide set of regulations issued by several Swiss entities.

\section{RESULTS}

\subsection{Ontologies}

We have defined several ontologies for representing the rules as well as the vocabulary (objects, spatial relations, geometry) emerging in these rules. These ontologues are interconnected, between them and with standards such as CityGML or IFC that exist in an ontological form.

\subsubsection{Rule expression}

The general model has the following main components (Figure 2a) and is detailed in (Caselli et al, 2020):

\section{- Rule}

- Condition, organized in Precondition and Postcondition, models the circumstances under which a rule is applicable (Precondition) and the implication it entails (Postcondition)

- Validity context, represents the temporal and/or spatial context within which the rule applies

- Object, represents an object as defined in the following ontologies (see 4.1.2 and 4.1.3) 
- Expression, expressed as Operand Operator Operand, represents a condition. An operand can be an expression or a value

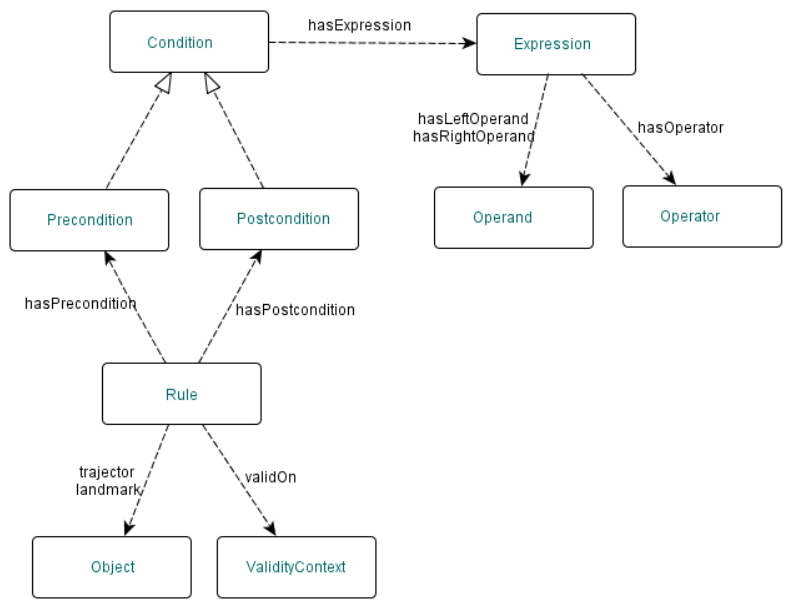

Figure 2a. Main components of the rule model

Figure $2 \mathrm{~b}$ gives and a more detailed view of the expression part:

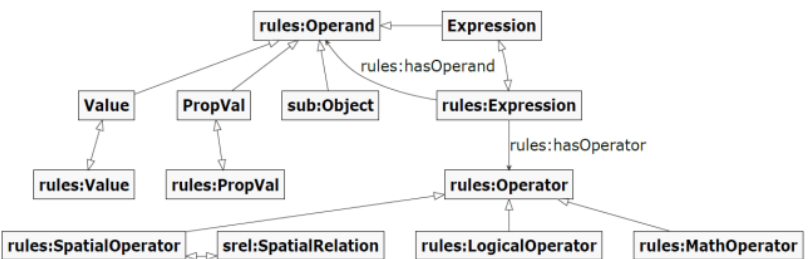

Figure 2b. Detailed view of the rule expression

For defining specific rules these components must be instantiated with elements defined in the following ontologies.

\subsubsection{Subsurface objects}

The subsurface objects are defined as subclasses of the CityGML class CityObject. They have been organized in manmade objects (underground buildings or building parts, tunnels, utility networks...) and natural objects (tree roots, groundwater, ...). A utility network is defined with nodes and links as in the CityGML Utility Network ADE.

\subsubsection{City objects}

Some rules or some subsurface objects relate to objects named City objects, that are on or above the ground and that can be found in CityGML. For example, a tree root relates to a tree which can be defined as subclass of SolitaryVegetationObject from CityGML. A gas pipe has to be placed under a sidewalk (and not under a car traffic area) which is a subclass of TrafficArea (from CityGML) Thus a tree or a sidewalk inherits the properties of the associated classes in CityGML, in particular a gml geometry.

\subsubsection{Spatial relations}

This ontology is not intended to represent in an exhaustive way all the spatial relations but those that can appear in compliance rules. Starting from the general ontology of spatial relations defined by Bucher et al (2012) and from compliance rules, we identified spatial relations that we organized in three main categories: topological relations (contains, disjoint, touches...), directional relations (above, below...) and distance relations (horizontal, vertical and direct distance). When we formulate rules, we choose in the ontology the relevant spatial relation, which corresponds to the semantics that we want and can be very specific in certain cases.

\subsubsection{Geometry}

Different types of geometry have been defined in this ontology since the compliance tests are performed with a solid geometry approach while the available data are represented as WKT geometry. Objects are associated to a solid geometry that we defined as a simplified version of the IFC solid geometry while being connected to this geometry. For example, the class BuildingOrBuildingPart is associated to a solid geometry which is a VExtrudedPolygon (an ExtrudedPolygon with a vertical extrusion direction). A VextrudedPolygon is defined by an outerSweptSurface (that will be extruded), a directrix of extrusion and a depth of extrusion. It is associated to an IfcExtrudedAreaSolid and to a WKT PolygonZ (see Figure 3).
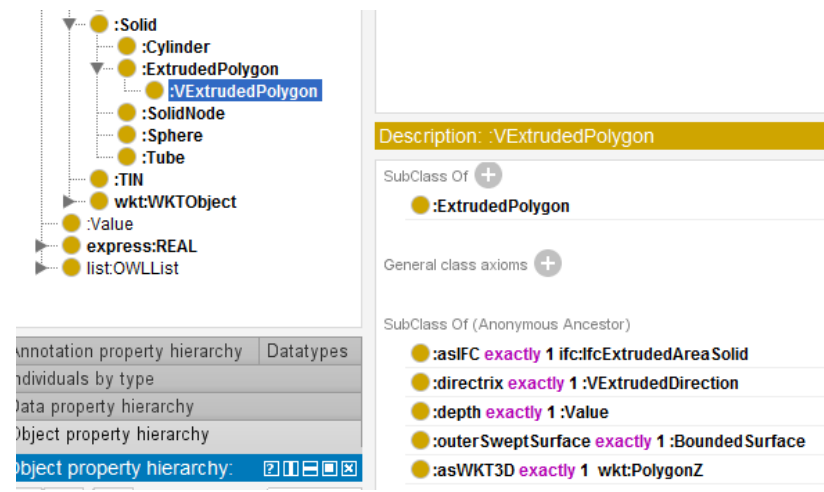

Figure 3. Part of the geometry ontology

Since all objects are defined as CityGML objects or subclasses of CityGML objects they also inherit the gml geometry defined in CityGML.

These ontologies are interconnected as illustrated in Figure 4. Arrows without labels represent subclass relations, either in the same ontology or from an ontology to another one.

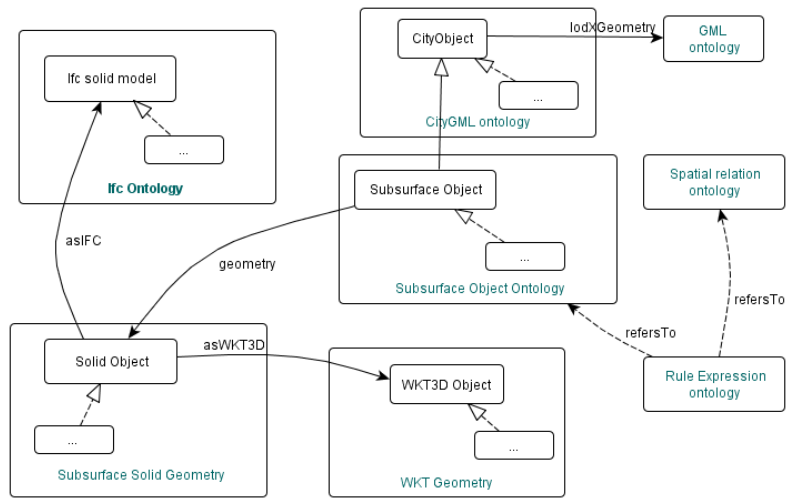

Figure 4. Ontologies architecture and interconnection

\subsection{Example}

The expression of the rule "a gas pipe must pass more than 2 meters from a tree root" can be represented (simplified) as illustrated in Figure 5. The distance is measured horizontally. 


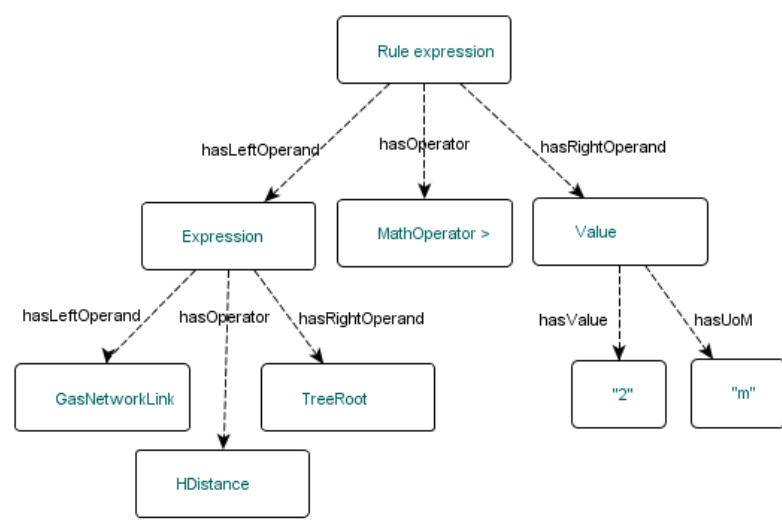

Figure 5. Expression of a specific rule

The previous rule expressed in Turtle is given below:

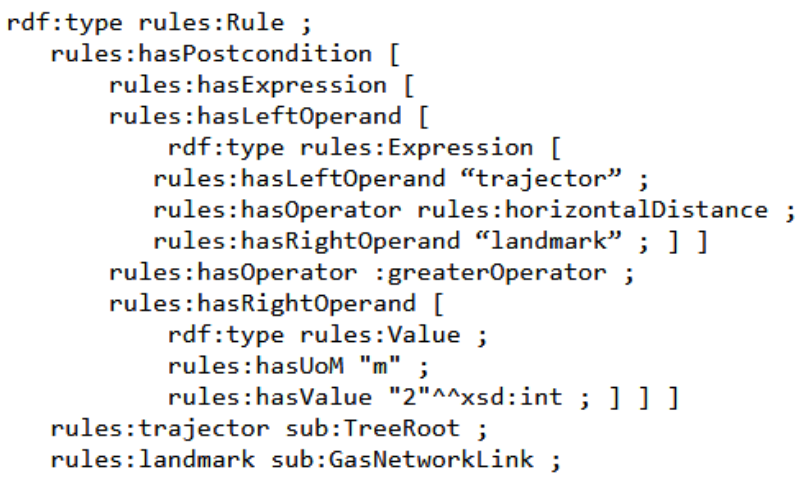

\section{CONCLUSION AND FUTURE WORK}

This paper presents an expressive model for representing compliance rules related to subsurface objects. Its expressiveness comes from its anchoring in a set of ontologies that describe (subsurface) city objects, their geometry and their semantic relationships. Rules expressed in this model can be automatically evaluated (with SHACL or SPARQL) on existing 3D city models expressed in RDF. We are currently developing a domain specific language to facilitate the expression of the rules by domain experts who are not IT specialists. This approach is complementary to the natural language processing approach to rule evaluation and best suited for complex rules (or complex written rules). We are also extending the model to transform compliance rules into completion rules that can infer missing data.

\section{REFERENCES}

Biljecki F., Kumar K. and Nagel C. (2018) CityGML Application Domain Extension (ADE): overview of developments. Biljecki et al. Open Geospatial Data, Software and Standards (2018) 3:13

Bucher B, Falquet G, Clementini E, Sester M (2012) Towards a typology of spatial relations and properties for urban applications. Usage, Usability, and Utility of $3 D$ City Models, 02010

Caselli A, Daponte V, Falquet G, Métral C (2020) A Rule Language Model for Subsurface Data Refinement. In: EG-ICE 2020 Workshop on Intelligent Computing in Engineering, pp. 443-452

Clementini E, Laurini R (2008) Un cadre conceptuel pour modéliser les relations spatiales. $R S$ 2008, vol. RNTI-E-14, pp. $1-17$

Gayathri R., Uma V. 2018. Ontology based knowledge representation technique, domain modeling languages and planners for robotic path planning: A survey. ICT Express 4 (2018) 69-74

Kalogianni E.; Dimopoulou E.; Quak W.; Germann M.; Jenni L.; Van Oosterom P (2017) INTERLIS Language for Modelling Legal 3D Spaces and Physical 3D Objects by Including Formalized Implementable Constraints and Meaningful Code Lists. In: ISPRS International Journal of Geo-Information, 6 (10), 319

Pauwels P. and Zhang S. (2015) Semantic rule-checking for regulation compliance checking: An overview of strategies and approaches. In Proceedings of the 32nd CIB W78 Conference, pp. 619-628

Wagner D., Wewetzer M., Bogdahn J., Alam N., Pries M. and Coors V. (2013) Geometric-Semantical ConsistencyValidation of CityGML Models. In: J. Pouliot et al. (eds), Progress and New Trends in 3D Geoinformation Sciences, Lecture Notes in Geoinformation and Cartography, Springer-Verlag Berlin Heidelberg, pp. 171-192

Xu X., Cai H. 2020. Semantic approach to compliance checking of underground utilities. Automation in construction 109 (2020) 103006

Revised July 2020

\section{ACKNOWLEDGEMENTS}

This research is supported by Innosuisse in the framework of the Innovation project 35265.1 IP-ICT, "Impulse-Subsurface: Efficient data exploitation in urban subsurface planning".

The authors would like to thank Ordnance Survey GB (https://www.ordnancesurvey.co.uk) and 1Spatial (https://1spatial.com/) for sponsoring the publication of this paper. 